\title{
APOE hypermethylation is associated with autism spectrum disorder in a Chinese population
}

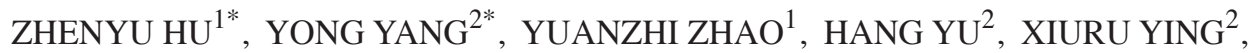 \\ DONGSHENG ZHOU ${ }^{1}$, JIE ZHONG ${ }^{2}$, ZHONGHUA ZHENG ${ }^{2}$, JING LIU ${ }^{2}$, RANRAN PAN ${ }^{2}$, \\ WENWU ZHANG ${ }^{1}$, FANG CHENG $^{1}$ and SHIWEI DUAN ${ }^{2}$
}

${ }^{1}$ Department of Child Psychiatry, Ningbo Kangning Hospital, Ningbo University; ${ }^{2}$ Zhejiang Provincial Key Laboratory of
Pathophysiology, Medical Genetics Center, School of Medicine, Ningbo University, Ningbo, Zhejiang 315211, P.R. China

Received November 13, 2017; Accepted March 1, 2018

DOI: $10.3892 /$ etm.2018.6069

\begin{abstract}
Abnormal apolipoprotein E (APOE) methylation has been demonstrated to be associated with Alzheimer's disease, which may have overlapping mechanisms with autism spectrum disorder (ASD). Thus, the purpose of the present study was to assess the possible link between APOE methylation and ASD. Genomic DNA was extracted from peripheral blood and subjected to a methylation assay. SYBR green-based quantitative methylation-specific polymerase chain reaction analysis was used to measure APOE methylation in 62 pediatric patients with ASD and 73 age-matched healthy subjects. The APOE methylation in each sample was expressed as a percentage of methylation of a reference (PMR). The results indicated that APOE methylation in pediatric patients with ASD was significantly higher than that in the healthy controls (median PMR, 33 vs. 11\%; $\mathrm{P}=2.36 \times 10^{-10}$ ). Receiver operating characteristic curve demonstrated that PMR of $15.4 \%$ was the optimal cut-off for predicting ASD (area under curve, 0.817; sensitivity, $93.5 \%$; specificity, $72.6 \%$; $\left.\mathrm{P}=2.36 \times 10^{-10}\right)$. In summary, the present results indicated that APOE hypermethylation in peripheral blood DNA may be used as a diagnostic biomarker for ASD.
\end{abstract}

\section{Introduction}

Autism spectrum disorder (ASD) comprises a range complex neurological diseases with impairments in social skills,

Correspondence to: Dr Shiwei Duan, Zhejiang Provincial Key Laboratory of Pathophysiology, Medical Genetics Center, School of Medicine, Ningbo University, 818 Fenghua Road, Jiangbei, Ningbo, Zhejiang 315211, P.R. China

E-mail: duanshiwei@nbu.edu.cn

\section{${ }^{*}$ Contributed equally}

Key words: apolipoprotein E, autism spectrum disorder, DNA methylation, peripheral blood, male communication and repetitive behaviors (1). The incidence of ASD in males is four times of that in females (2). ASD causes great obstacles in interpersonal relationships (3), and it has become a heavy burden on society and the families of affected individuals $(4,5)$. Early intervention may change the long-term prognosis of ASD patients (6).

The pathogenesis of ASD involves a complex interaction between heredity and environment (7). Epigenetics may provide the best bridge between genetic and environmental factors (8). DNA methylation is an important epigenetic modification that regulates the expression of numerous functional genes in the human nervous system $(6,9)$. Aberrant DNA methylation has been reported in diseases or disorders of the central nervous system $(10,11)$. These include hypermethylation of oxytocin R (12), Reelin (13), and SH3 and multiple ankyrin repeat domains 3 (14) in ASD.

Apolipoprotein E (APOE) encodes a glycoprotein associated with lipoproteins in the periphery and the brain $(15,16)$. Abnormal methylation of APOE was reported to be associated with Alzheimer's disease (AD) (17), which may have overlapping mechanisms with ASD (18). A genetic study indicated that a variant of APOE is associated with ASD (19); however, it has remained elusive whether APOE methylation is linked to ASD.

In the light of those previous results, the goal of the present study was to explore whether APOE methylation is associated with ASD.

\section{Materials and methods}

Patients and samples. A total of 135 pediatric subjects, including 62 ASD patients (with no other disorders or diseases) and 73 healthy age-matched individuals, volunteered for the study and were recruited at Ningbo Kangning Hospital (Ningbo, China) between September 2015 and September 2017. The ASD patients were diagnosed according to the Diagnostic and Statistical Manual of Mental Disorders, Fourth Edition, the Autism Diagnostic Observation Schedule and the Childhood Autism Rating Scales (CARS) (5). Blood samples were drawn from healthy controls and ASD cases prior to treatment. The clinical data acquired from patients mainly included maternal pregnancy history, family history, and disease duration. The 
study was approved by the Ethics Committees in Ningbo Kangning Hospital and Ningbo University (Ningbo, China), and written informed consent was obtained from the parents of all patients.

DNA isolation and bisulphite conversion. DNA was extracted from blood with the E.Z.N.A. ${ }^{\mathrm{TM}}$ Blood DNA Kit (Omega Bio-Tek, Norcross, GA, USA), according to the manufacturer's instructions. The Nanodrop2000 spectrophotometer (Thermo Fisher Scientific, Inc., Waltham, MA, USA) was used to measure the DNA concentration. The EZ DNA Methylation-Gold kit ${ }^{\mathrm{TM}}$ (Zymo Research, Irvine, CA, USA) was used to convert unmethylated cytosines into the corresponding uracils, while the methylated cytosines remained in their positions.

SYBR green-based quantitative methylation-specific polymerase chain reaction ( $q M S P$ ). qMSP was used for to assess the methylation of APOE. The reaction system contained $10 \mu 1$ SYBR Green I Master Mix (Roche Diagnostics, Basel, Switzerland), $0.5 \mu 1$ forward primer $(10 \mu \mathrm{m}), 0.5 \mu \mathrm{l}$ reverse primer $(10 \mathrm{~m}), 1.0 \mu \mathrm{l}$ templates and $8 \mu \mathrm{l}$ double-distilled (dd) $\mathrm{H}_{2} \mathrm{O}$. The thermocycling conditions comprised an initial denaturation at $95^{\circ} \mathrm{C}$ for $10 \mathrm{~min}$, followed by 45 cycles of $95^{\circ} \mathrm{C}$ for $30 \mathrm{sec}, 58^{\circ} \mathrm{C}$ for $30 \mathrm{sec}$ and $72^{\circ} \mathrm{C}$ for $30 \mathrm{sec}$. Subsequently, melting curve analysis was performed at $95^{\circ} \mathrm{C}$ for $15 \mathrm{sec}$ and $60^{\circ} \mathrm{C}$ for $1 \mathrm{~min}$, followed by increases in the temperature by $0.11^{\circ} \mathrm{C}$ per sec to $95^{\circ} \mathrm{C}$. Human sperm DNA (Beijing Solarbio Science \& Technology Co., Ltd., Beijing, China) was methylated as the positive control by excess SssI methyltransferase (Thermo Fisher Scientific, Inc.), and $\mathrm{ddH}_{2} \mathrm{O}$ as a negative control. ACTB was used as the internal reference to correct the differences in the quality and quantity between samples. The sequences of the qMSP primers were APOE forward, 5'-CGAGGTGTA GGTTATGTTC-3' and reverse, 5'-TACGCAACTTACGCA AAT-3'; and $\beta$-actin (ACTB) forward, 5'-TGGTGATGG AGGAGGTTTAGTAAGT-3' and reverse, 5'-AACCAA TAAAACCTACTCCTCCCTTAA-3'. The percentage of methylation of a reference (PMR) of APOE in each sample was calculated by the following approach: $\Delta \Delta \mathrm{Cq}=$ sample DNA $\left(\mathrm{Cq}_{\mathrm{APOE}}\right.$ gene- $-\mathrm{Cq}_{\mathrm{ACTB}}$ control $)$-fully methylated DNA $\left(\mathrm{Cq}_{\mathrm{APOE} \text { gene }}-\mathrm{Cq}_{\mathrm{ACTB} \text { control }}\right)(20,21)$.

Statistical analysis. All statistical analyses were performed using SPSS software version 18.0 (SPSS, Inc., Chicago, IL, USA). The independent-samples t-test was applied for the comparisons of differences in APOE methylation between ASD cases and controls. Receiver operating characteristic curves (ROC) were drawn to evaluate the diagnostic value of APOE methylation for ASD. The Spearman rank test was applied to assess the associations between APOE methylation and clinical indicators in ASD. $\mathrm{P}<0.05$ was considered to indicate a statistically significant difference.

\section{Results}

As presented in Fig. 1A, a methylation assay was performed on an 88-bp fragment of the $\mathrm{CpG}$ island of APOE. A successful bisulphite transformation was evidenced by detection of transformed thymine by Sanger sequencing (Fig. 1B). The target fragment length of 88 bp was confirmed by capillary electrophoresis (Fig. 1C).

The present results indicated that APOE methylation in pediatric ASD patients was significantly higher than that in healthy age-matched individuals (median PMR, 33.36 vs. $10.61 \% ; \mathrm{P}=2.36 \times 10^{-10} ;$ Fig. $2 \mathrm{~A}$ ). APOE hypermethylation was identified in 36 out of 62 subjects with ASD. Considering the higher prevalence of ASD in males, a breakdown analysis by gender was performed. The results indicated that APOE methylation levels were higher in male ASD cases than in healthy male controls (median PMR, 32.12 vs. $11.00 \%$; $\mathrm{P}=8.51 \times 10^{-7}$; Fig. $\left.2 \mathrm{~B}\right)$. Similar results were also obtained in females (mean PMR, 43.35 vs. $7.87 \%$; $\mathrm{P}=4.18 \times 10^{-6}$; Fig. 2C).

In addition, ROC curve analysis was performed to quantitatively evaluate the diagnostic value. As presented in Fig. 3A, the area under the curve (AUC) was 0.817 [95\% confidence interval (CI), 0.741-0.893]. The ROC curves demonstrated that PMR of $15.4 \%$ was the optimal cut-off for predicting ASD (sensitivity, 93.5\%, specificity, $72.6 \% ; \mathrm{P}=2.36 \times 10^{-10}$; Fig. 3A). Further subgroup analysis by gender provided a similar result in males $(\mathrm{AUC}=0.772$; 95\% CI, 0.682-0.865; sensitivity, 93.9\%; specificity, 67.7\%; $\mathrm{P}=8.52 \times 10^{-7}$; Fig. 3B), PMR of $15.3 \%$ was the optimal cut-off for predicting ASD. Due to a limited number of female individuals, it was not possible to perform any ROC curve analysis in females.

\section{Discussion}

APOE is a multifunctional protein in the brain, and it is known as a carrier of cholesterol and other lipids in the central nervous system (22-24). APOE methylation levels have been reported to be significantly elevated in AD patients (17). Previous studies have indicated that ASD and AD have various common mechanisms in the course of their development (25-27). Therefore, it was speculated that APOE is associated with the occurrence and the development of ASD. The present results demonstrated that APOE hypermethylation is significantly associated with ASD. Based on the ROC curve, APOE hypermethylation may be regarded as a potential biomarker for the diagnosis of ASD.

APOE polymorphisms (APOE $\varepsilon 2$ and APOE $\varepsilon 4$ ) are involved in the etiological complexity of the predisposition for ASD $(19,28)$. A transmission distortion of the APOE $\varepsilon 2$ allele has been reported in families with cases of ASD. Compared with APOE $\varepsilon 3$ and APOE $\varepsilon 4$, the APOE $\varepsilon 2$ protein product displays the lowest receptor binding affinity (28). DNA methylation is an epigenetic modification and regulates gene expression in response to environmental stimuli (29). Gene methylation is generally inversely correlated with gene expression (30-32). APOE expression has been reported to be downregulated by APOE hypermethylation in malignant transformed TRL 1215 cells (33). Due to material restrictions, it was not possible to measure the correlation between APOE expression and APOE methylation in the present study. Thus, The Cancer Genome Atlas (TCGA) database was used to collect the methylation and the transcription data of APOE. This data analysis in TCGA indicated that APOE hypermethylation was indeed inversely correlated with lower expression $\left(\mathrm{P}=1.96 \times 10^{-27}, \mathrm{r}=-0.604\right)$. 



Figure 1. Characteristics of target sequences in the APOE gene. (A) APOE is located on chr19 (q13.32) and the 80-bp target fragment is located on the CpG island of APOE. (B) Sanger sequencing verified a successful bisulfite conversion. The upper row represents the original sequence and the lower row represents the converted sequence. (C) Results of capillary electrophoresis. MARKER denotes the DNA ladder marker and BLANK denotes an experimental negative control. The numbers on the left indicate the number of bps of the DNA ladder markers. The target fragment length was $88 \mathrm{bp}$. Chr, chromosome; APOE, apolipoprotein E; F, forward; R, Reverse; Seq, sequencing; UCSC, University of California, Santa Cruz; SNP, single nucleotide polymorphism.
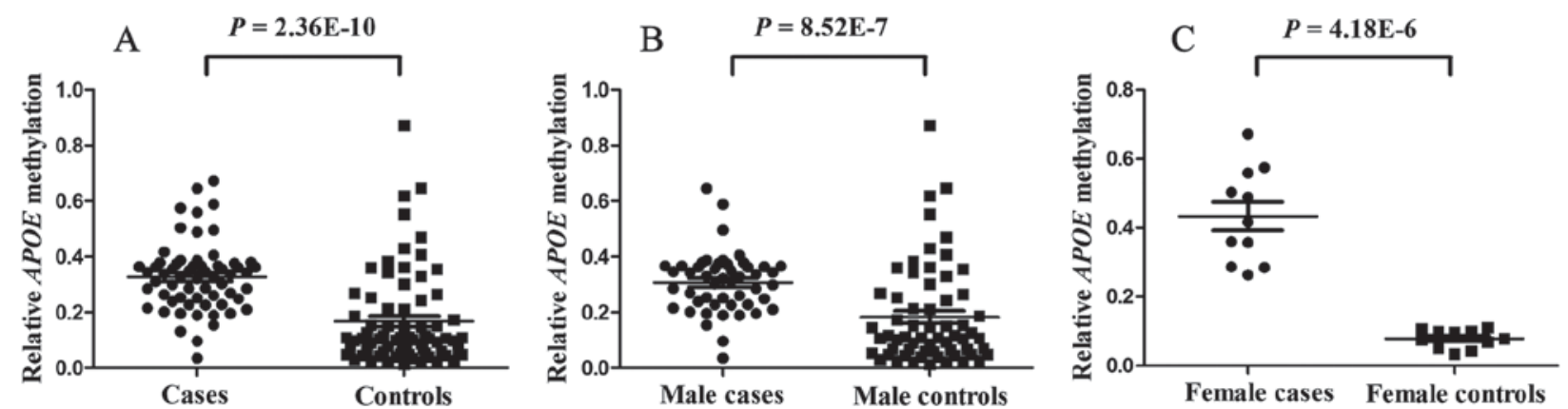

Figure 2. Comparison of the relative APOE methylation between ASD cases and healthy controls. (A) Comparison between ASD cases and controls. The median PMR in cases and controls was 33\% (interquartile range, 25 and 37\%) and 11\% (interquartile range, 6 and 23\%), respectively. The P-value for the comparison between the cases and the controls was $2.36 \times 10^{-10}$. (B) Comparison between male ASD cases and male controls. The median PMR values of cases and controls were $32 \%(23,37 \%)$ and $11 \%(6,27 \%)$, respectively. The P-value for the comparison between the male cases and the male controls was $8.52 \times 10^{-10}$. (C) Comparison between female ASD cases and female controls. The median PMR values of cases and controls were $0.42(0.29,0.56)$ and $0.08(0.05,0.10)$, respectively. The P-value for the comparison between the female cases and the female controls was $4.18 \times 10^{-6}$. APOE, apolipoprotein E; ASD, autism spectrum disorder; PMR, percentage of methylation of a reference. 
A

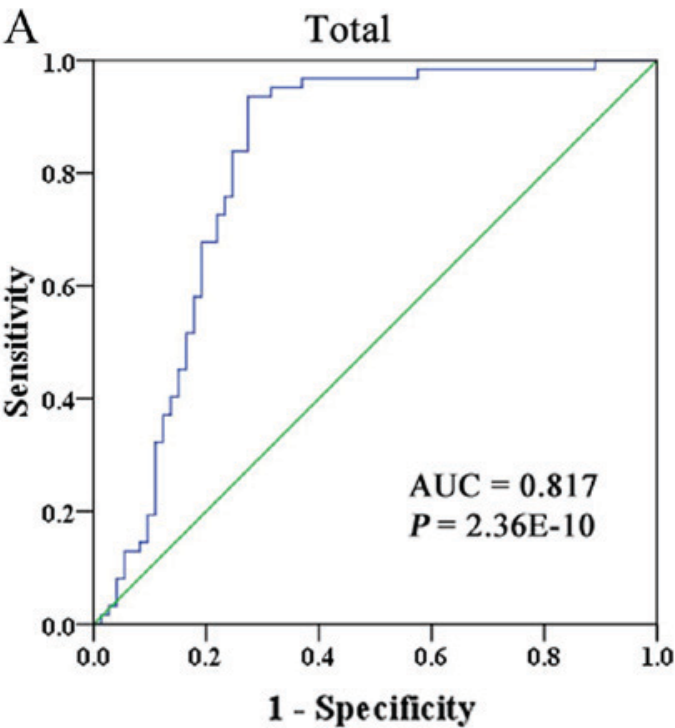

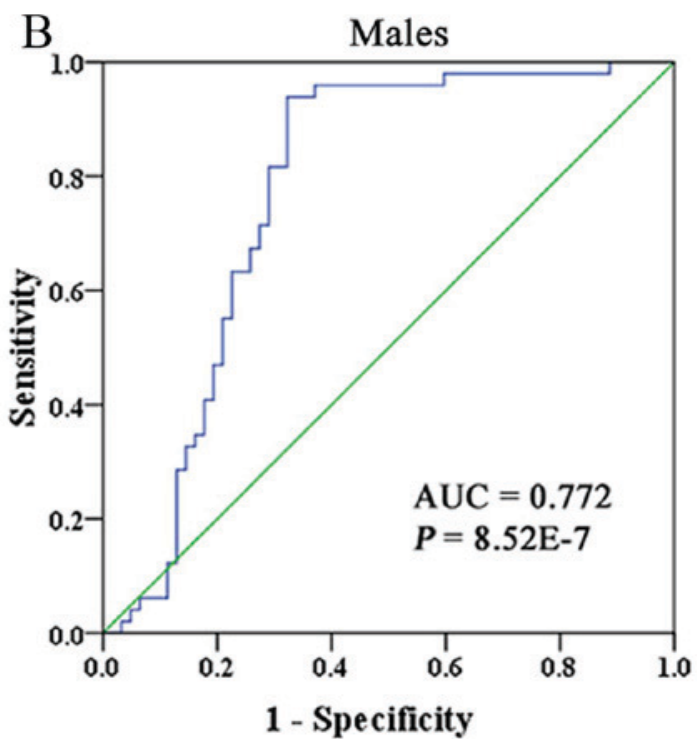

Figure 3. ROC curve of APOE methylation for the diagnosis of ASD. The ROC curve was generated to demonstrate the sensitivity (Y-axis) and the specificity (X-axis) of the continuous indicators. (A) A ROC curve was used to estimate the diagnostic value of APOE methylation for ASD. The AUC was 0.817 (95\% CI: $0.741-0.893$ ) with a sensitivity of $93.5 \%$ and a specificity of $72.6 \%$ when the cut-off was $15.4 \%$. (B) ROC curve analysis in the male sub-population $(\mathrm{AUC}=0.773$; 95\% CI, 0.682-0.865; sensitivity, 93.9\%; specificity, 67.7\%). AUC, area under the curve; ROC, receiver operating characteristic; APOE, apolipoprotein E; ASD, autism spectrum disorder.

In the present case-control study, APOE hypermethylation was detected in pediatric patients with ASD compared with healthy age-matched individuals. Therefore, it was speculated that APOE hypermethylation may reduce APOE expression, eventually leading to the onset of ASD.

Early intervention of ASD may change the long-term prognosis of ASD patients (6). The current diagnostic methods for ASD comprise the ASD behavior checklist (ABC) (34), the CARS (5) and the Social Communication Questionnaire (SCQ) (35). The ABC scale has a specificity of $97 \%$ and a sensitivity of $38 \%$ with an idiomatic cutoff score at 67 (36). In addition, the CARS has a sensitivity of $83 \%$ and a specificity of $82 \%$ (37), and the SCQ scale has a sensitivity of $85 \%$ and a specificity of $75 \%$ (35). These tests are also substantially used in the clinic.

A large variety of molecular biomarkers have been studied for their diagnostic value in ASD, including paraoxonase-1 (PON-1), lipoprotein-associated phospholipase A2 (Lp-PLA2) (38), microRNAs $(39,40)$, interleukin (IL)-6 and tumor necrosis factor (TNF) $\alpha$ (41). However, only few biomarkers are actually used as an auxiliary in the diagnosis of ASD in the clinic. The sensitivity of Lp-PLA2 is $70 \%$ and its specificity is 77\%; furthermore, $\mathrm{PON}-1$ has a relatively low AUC of 0.660 (sensitivity, 59\%; specificity, 63\%) (38). IL- 6 and TNF $\alpha$ have a low sensitivity of 84.0 and $76.0 \%$, respectively, for the diagnosis of ASD (41). In the present study, APOE methylation yielded a higher AUC of 0.817 (sensitivity, 93.5\%; specificity, 72.6\%). It is possible to accurately measure DNA methylation in a variety of materials, including plasma and serum, and it is considered an ideal marker for experimental determination $(42,43)$. The results of the present study indicated that APOE methylation has a high diagnostic value for ASD, suggesting that APOE hypermethylation in peripheral blood may be a biomarker for the early diagnosis of ASD.
In conclusion, the present study indicated that APOE hypermethylation is associated with ASD. Future studies may be necessary to confirm the utility of APOE hypermethylation in peripheral blood DNA as a diagnostic marker for ASD.

\section{Acknowledgements}

Not applicable.

\section{Funding}

The present study was supported by the Medical Science and Technology Project of Zhejiang Province (grant no. 2017207569) and the K.C. Wong Magna Fund of Ningbo University.

\section{Availability of data and materials}

The analyzed data sets generated during the study are available from the corresponding author on reasonable request.

\section{Authors' contributions}

ZH, YY and SD contributed to the conception, design and final approval of the submitted version. YZ, HY, XY, DZ, JZ, ZZ, JL, RP, WZ and FC contributed by performing the experiments, interpreting the data and designing the figures. All authors read and approved the final manuscript.

\section{Ethical approval and consent to participate}

The study was approved by the Ethics Committees in Ningbo Kangning Hospital and Ningbo University and written informed consent was obtained from the parents of all the children. 


\section{Consent for publication}

Written informed consent was obtained for the publication of the patient's data.

\section{Competing interests}

The authors declare that they have no competing interests.

\section{References}

1. Shpyleva S, Ivanovsky S, de Conti A, Melnyk S, Tryndyak V, Beland FA, James SJ and Pogribny IP: Cerebellar oxidative DNA damage and altered DNA methylation in the BTBR T+tf/J mouse model of autism and similarities with human post mortem cerebellum. PLoS One 9: e113712, 2014.

2. Ginsberg MR, Rubin RA, Falcone T, Ting AH and Natowicz MR: Brain transcriptional and epigenetic associations with autism. PLoS One 7: e44736, 2012

3. Bird G and Cook R: Mixed emotions: The contribution of alexithymia to the emotional symptoms of autism. Transl Psychiatry 3: e285, 2013

4. Buescher AV, Cidav Z, Knapp M and Mandell DS: Costs of autism spectrum disorders in the United Kingdom and the United States. JAMA Pediatr 168: 721-728, 2014.

5. Howsmon DP, Kruger U, Melnyk S, James SJ and Hahn J: Classification and adaptive behavior prediction of children with autism spectrum disorder based upon multivariate data analysis of markers of oxidative stress and DNA methylation. PLoS Comput Biol 13: e1005385, 2017.

6. Fernell E, Eriksson MA and Gillberg C: Early diagnosis of autism and impact on prognosis: A narrative review. Clin Epidemiol 5: 33-43, 2013

7. Chen T, Giri M, Xia Z, Subedi YN and Li Y: Genetic and epigenetic mechanisms of epilepsy: A review. Neuropsychiatr Dis Treat 13: 1841-1859, 2017

8. Esteller M: Profiling aberrant DNA methylation in hematologic neoplasms: A view from the tip of the iceberg. Clin Immunol 109: 80-88, 2003

9. Mill J,Tang T,KaminskyZ, Khare T, Yazdanpanah S, BouchardL, Jia P, Assadzadeh A, Flanagan J, Schumacher A, et al: Epigenomic profiling reveals DNA-methylation changes associated with major psychosis. Am J Hum Genet 82: 696-711, 2008.

10. Cheng J, Wang Y, Zhou K, Wang L, Li J, Zhuang Q, Xu X, Xu L, Zhang K, Dai D, et al: Male-specific association between dopamine receptor D4 gene methylation and schizophrenia. PLoS One 9: e89128, 2014

11. Dai D, Cheng J, Zhou K, Lv Y, Zhuang Q, Zheng R, Zhang K, Jiang D, Gao S and Duan S: Significant association between DRD3 gene body methylation and schizophrenia. Psychiatry Res 220: 772-777, 2014

12. Elagoz Yuksel M, Yuceturk B, Karatas OF, Ozen M and Dogangun B: The altered promoter methylation of oxytocin receptor gene in autism. J Neurogenet 30: 280-284, 2016.

13. Lintas C, Sacco R and Persico AM: Differential methylation at the RELN gene promoter in temporal cortex from autistic and typically developing post-puberal subjects. J Neurodev Disord 8: 18, 2016.

14. Zhu L, Wang X, Li XL, Towers A, Cao X, Wang P, Bowman R, Yang H, Goldstein J, Li YJ and Jiang YH: Epigenetic dysregulation of SHANK3 in brain tissues from individuals with autism spectrum disorders. Hum Mol Genet 23: 1563-1578, 2014.

15. Tamboli IY, Heo D and Rebeck GW: Extracellular proteolysis of apolipoprotein $\mathrm{E}$ (apoE) by secreted serine neuronal protease. PLoS One 9: e93120, 2014.

16. Raiford KL, Shao Y,Allen IC,MartinER, MenoldMM, WrightHH, Abramson RK, Worley G, DeLong GR, Vance JM, et al: No association between the APOE gene and autism. Am J Med Genet B Neuropsychiatr Genet 125B: 57-60, 2004.

17. Foraker J, Millard SP, Leong L, Thomson Z, Chen S, Keene CD, Bekris LM and Yu CE: The APOE gene is differentially methylated in Alzheimer's disease. J Alzheimers Dis 48: 745-755, 2015

18. Napoli E, Ross-Inta C, Wong S, Hung C, Fujisawa Y, Sakaguchi D Angelastro J, Omanska-Klusek A, Schoenfeld R and Giulivi C: Mitochondrial dysfunction in Pten haplo-insufficient mice with social deficits and repetitive behavior: Interplay between Pten and p53. PLoS One 7: e42504, 2012.
19. Giunco CT, de Oliveira AB, Carvalho-Salles AB, Souza DS, Silva AE, da Rocha SS and Fett-Conte AC: Association between APOE polymorphisms and predisposition for autism. Psychiatr Genet 19: 338, 2009

20. Kristensen LS, Mikeska T, Krypuy M and Dobrovic A: Sensitive melting analysis after real time-methylation specific PCR (SMART-MSP): High-throughput and probe-free quantitative DNA methylation detection. Nucleic Acids Res 36: e42, 2008.

21. Chen X, Hu H, Liu J, Yang Y, Liu G, Ying X, Chen Y, Li B, Ye C, Wu D and Duan S: FOXF2 promoter methylation is associated with prognosis in esophageal squamous cell carcinoma. Tumour Biol 39: 1010428317692230, 2017.

22. Huang Y and Mahley RW: Apolipoprotein E: Structure and function in lipid metabolism, neurobiology, and Alzheimer's diseases. Neurobiol Dis 72: 3-12, 2014

23. Liu S, Park S, Allington G, Prelli F, Sun Y, Sun Y, Martá-Ariza M, Scholtzova H, Biswas G2, Brown B, Verghese PB, et al: Targeting Apolipoprotein E/Amyloid $\beta$ Binding by Peptoid CPO_A $\beta 17-21$ $\mathrm{P}$ Ameliorates Alzheimer's disease related pathology and cognitive decline. Sci Rep 7: 8009, 2017

24. Zhao N, Liu CC, Qiao W and Bu G: Apolipoprotein E, Receptors, and Modulation of Alzheimer's Disease. Biol Psychiatry 83: 347-357, 2018.

25. Folmsbee SS, Wilcox DR, Tyberghein K, De Bleser P, Tourtellotte WG, van Hengel J, van Roy F and Gottardi CJ: $\alpha \mathrm{T}$-catenin in restricted brain cell types and its potential connection to autism. J Mol Psychiatry 4: 2, 2016.

26. Blomqvist ME, Andreasen N, Bogdanovic N, Blennow $\mathrm{K}$, Brookes AJ and Prince JA: Genetic variation in CTNNA3 encoding alpha-3 catenin and Alzheimer's disease. Neurosci Lett 358: 220-222, 2004.

27. Martin ER, Bronson PG,Li YJ, Wall N, Chung RH, SchmechelDE, Small G, Xu PT, Bartlett J, Schnetz-Boutaud N, et al: Interaction between the alpha-T catenin gene (VR22) and APOE in Alzheimer's disease. J Med Genet 42: 787-792, 2005.

28. Persico AM, D'Agruma L, Zelante L, Militerni R, Bravaccio C, Schneider C, Melmed R, Trillo S, Montecchi F, Elia M, et al: Enhanced APOE2 transmission rates in families with autistic probands. Psychiatr Genet 14: 73-82, 2004.

29. Jaenisch R and Bird A: Epigenetic regulation of gene expression: How the genome integrates intrinsic and environmental signals. Nat Genet 33 (Suppl): S245-S254, 2003.

30. Tamura Y, Kunugi H, Ohashi J and Hohjoh H: Epigenetic aberration of the human REELIN gene in psychiatric disorders. Mol Psychiatry 12: 519, 593-600, 2007.

31. Zilberman D, Gehring M, Tran RK, Ballinger T and Henikoff S: Genome-wide analysis of Arabidopsis thaliana DNA methylation uncovers an interdependence between methylation and transcription. Nat Genet 39: 61-69, 2007.

32. Zhang X, Yazaki J, Sundaresan A, Cokus S, Chan SW, Chen H, Henderson IR, Shinn P, Pellegrini M, Jacobsen SE and Ecker JR: Genome-wide high-resolution mapping and functional analysis of DNA methylation in arabidopsis. Cell 126: 1189-1201, 2006.

33. Suzuki M, Takeda S, Teraoka-Nishitani N, Yamagata A, Tanaka T, Sasaki M, Yasuda N, Oda M, Okano T, Yamahira K, et al: Cadmium-induced malignant transformation of rat liver cells: Potential key role and regulatory mechanism of altered apolipoprotein $\mathrm{E}$ expression in enhanced invasiveness. Toxicology 382 : $16-23,2017$.

34. Juneja M, Sharma S and Mukherjee SB: Sensitivity of the autism behavior checklist in Indian autistic children. J Dev Behav Pediatr 31: 48-49, 2010.

35. Dykens EM, Roof E, Hunt-Hawkins H, Dankner N, Lee EB, Shivers CM, Daniell C and Kim SJ: Diagnoses and characteristics of autism spectrum disorders in children with Prader-Willi syndrome. J Neurodev Disord 9: 18, 2017.

36. Nordin V and Gillberg C: Autism spectrum disorders in children with physical or mental disability or both. II: Screening aspects. Dev Med Child Neurol 38: 314-324, 1996.

37. García-López C and Narbona J: Clinical usefulness of IDEA and CARS: Concordance with DSM-IV-TR in children and adolescents with suspicion of PDD. An Pediatr (Barc) 80: 71-76, 2014 (In Spanish).

38. Hayek J, Cervellati C, Crivellari I, Pecorelli A and Valacchi G: Lactonase activity and lipoprotein-phospholipase A2 as possible novel serum biomarkers for the differential diagnosis of autism spectrum disorders and rett syndrome: Results from a pilot study. Oxid Med Cell Longev 2017: 5694058, 2017. 
39. Mundalil Vasu M, Anitha A, Thanseem I, Suzuki K, Yamada K Takahashi T, Wakuda T, Iwata K, Tsujii M, Sugiyama T and Mori N: Serum microRNA profiles in children with autism. Mol Autism 5: 40, 2014.

40. Hicks SD, Ignacio C, Gentile K and Middleton FA: Salivary miRNA profiles identify children with autism spectrum disorder, correlate with adaptive behavior, and implicate ASD candidate genes involved in neurodevelopment. BMC Pediatr 16: 52, 2016.

41. El-Ansary AK, Ben Bacha AG and Al-Ayadhi LY: Proinflammatory and proapoptotic markers in relation to mono and di-cations in plasma of autistic patients from Saudi Arabia. J Neuroinflammation 8: 142, 2011
42. Wu P, Cao Z and Wu S: New progress of epigenetic biomarkers in urological cancer. Dis Markers 2016: 9864047, 2016.

43. Tsui NB, Ng EK and Lo YM: Stability of endogenous and added RNA in blood specimens, serum, and plasma. Clin Chem 48: $1647-1653,2002$.

(7)(9) This work is licensed under a Creative Commons

cc) Attribution-NonCommercial-NoDerivatives 4.0 International (CC BY-NC-ND 4.0) License. 